\title{
UNA EDUCACIÓN INTEGRAL PARA LOS MÉDICOS DE LA UNIVERSIDAD DE BUENOS AIRES: LAS PROPUESTAS DE JOSÉ RAMOS MEJÍA Y BERNARDO HOUSSAY ENTRE 1870 Y 1940
}

\author{
Pablo von Stecher \\ Instituto de Lingüística, Facultad de Filosofía y Letras, Universidad de Buenos Aires -CONICET. \\ pablovonstecher@gmail.com
}

Recibido: 1 agosto 2014; Aceptado: 9 enero 2015.

Cómo citar este artículo/Citation: Stecher, Pablo von (2016), “Una educación integral para los médicos de la Universidad de Buenos Aires: las propuestas de José Ramos Mejía y Bernardo Houssay entre 1870 y 1940”, Asclepio 68 (1): p134. doi: http://dx.doi.org/10.3989/asclepio.2016.13

RESUMEN: Hacia fines del siglo XIX, algunos médicos argentinos que enseñaban en la Facultad de Medicina de la Universidad de Buenos Aires entendían que los estudiantes y futuros médicos debían ser formados en un saber integral, que superara las materias clínicas. De este modo, intentaron inculcarles el conocimiento de las letras y el arte, pero también promovieron las ventajas del desarrollo físico, forjado en las prácticas deportivas, necesario para fortalecer a los alumnos ante una carrera de tal magnitud y para acrecentar los sentimientos de confraternidad y camaradería. Hacia la década de 1920, Bernardo Houssay, célebre médico argentino y ganador del premio Nobel (en 1947), volvía a insistir sobre la necesidad de una educación integral del estudiante de medicina y proponía, para su materialización, el proyecto de una ciudad universitaria en Buenos Aires. Este texto analiza, entonces, el modo en que se buscó formar a los alumnos en diversas prácticas y conocimientos, más allá de los estrictamente médicos, e indaga los distintos proyectos (políticos, sociales) vinculados a tales propósitos que tuvieron lugar durante estas décadas en la Argentina.

PALABRAS CLAVE: Medicina; Enseñanza; Higienismo; Deportes; Literatura.

\section{AN INTEGRAL EDUCATION FOR THE DOCTORS OF THE UNIVERSIDAD DE BUENOS AIRES: THE SU- GGESTIONS OF JOSÉ RAMOS MEJÍA AND BERNARDO HOUSSAY BETWEEN 1870 AND 1940}

ABSTRACT: At the end of nineteenth century, certain Argentinean doctors who taught medicine at the Universidad de Buenos Aires considered that students had to develop an integral knowledge able to outweigh the medicine subjects. Consequently, not only have they tried to inculcate this wide knowledge into the students -which included literature and art concepts- but have also promoted the advantages of the physical activity -derived from sports- as necessary to strengthen the students, allowing them to face such extensive career and, in this way, increase the feelings of friendship and brotherhood. In 1920, Bernardo Houssay, renowned Argentinean doctor and Nobel Prize winner (in 1947), again insisted on the need of an exhaustive education and a harmonious development for the medicine student, for which he suggested building a University campus in Buenos Aires. Therefore, this paper analyzes the attempts of training the students in different practices and widening their knowledge beyond the strictly medical concepts. It also looks into the different projects (political, social) in connection with the purposes above mentioned which were supported during those years in Argentina.

KEY WORDS: Medicine; Teaching; Hygienism; Sports; Literature.

Copyright: () 2016 CSIC. Este es un artículo de acceso abierto distribuido bajo los términos de la licencia Creative Commons Attribution (CC BY) España 3.0. 


\section{INTRODUCCIÓN}

En la Facultad de Medicina de la Universidad de Buenos Aires, desde su fundación como Departamento independiente en 1821, han estudiado distintas figuras que se destacaron en el desarrollo de las ciencias clínicas en la Argentina, pero también en materia de sociología, política, educación e incluso literatura. Algunos de estos médicos, como Eduardo Wilde, José María Ramos Mejía, José Ingenieros, Héctor Taborda o Bernardo Houssay, que además se desempeñaron como profesores o jefes de cátedra, intentaron proyectar desde sus discursos académicos (lecciones, artículos, conferencias, discursos inaugurales de cátedra) un alumnado formado en una educación integral, cuyos conocimientos se ampliaran desde las materias clínicas a otro tipo de saberes, como podrían ser los de las letras, el arte y los idiomas. Se trataba, además, de una formación en la que confluyeran los beneficios físicos y sociales de los deportes y de las actividades fisicas. Sin conformarse como un proyecto homogéneo o estructurado, estas propuestas pueden rastrearse en distintos momentos desde la reincorporación de la Facultad de Medicina a la Universidad de Buenos Aires (en 1871) hasta las décadas de 1930 y 1940, momento de expansión discursiva de Bernardo Houssay, médico paradigmático de la investigación en fisiología en la Argentina y ganador del Premio Nobel en Medicina (en 1947). Nos proponemos, entonces, indagar el modo en que algunas voces médicas fundacionales de la ciencia y de la cultura argentina buscaron instruir a los estudiantes y futuros médicos de Buenos Aires y reflexionar sobre las distintas justificaciones e intencionalidades de sociabilidad médica y política subyacentes a tales procesos de educación.

\section{NOTAS SOBRE EL DESARROLLO DE LA MEDICINA EN BUENOS AIRES}

Las pioneras instituciones dedicadas a la instrucción clínica en Buenos Aires datan del año 1780, momento en que se crea el Protomedicato, ocupado de controlar la salud pública para también de enseñar la medicina (Buchbinder, 2005, p. 43). En tanto, la primera Escuela de Medicina y Cirugía en Buenos Aires fue inaugurada oficialmente en 1801 bajo un plan de seis años de duración. Luego, con la creación de la Universidad de Buenos Aires en 1821, se fundaba un Departamento de Medicina. El programa de los estudios médicos se establecía en este espacio a través de cinco cátedras: instituciones médicas, instituciones quirúrgicas, clínica médica, clínica quirúrgica y farmacia (Pérgola y Fustinoni, 1969, p. 81).
No obstante, en las décadas siguientes, el desarrollo de los estudios académicos atravesaría una etapa de oscuridad devenida de la reducida inversión intelectual y presupuestaria entre los años 1835 y 1852, bajo el gobierno federal de Juan Manuel de Rosas ${ }^{1}$. En este período se forzó tanto la renuncia de consagrados profesores, como el abandono voluntario de otros y el uso obligatorio del emblema rosista (la divisa punzó) entre los catedráticos, asimismo, se incrementaron los aranceles al tiempo que los libros de texto comenzaron a escasear. Sin embargo, a pesar del declive académico y científico de estos años, es certero afirmar que egresaron 168 estudiantes del Departamento de Medicina entre 1835 y 1852 (Pérgola y Fustinoni, 1969, pp. 80-84; Buchbinder, 2005, p. 43).

Resulta fundamental señalar que, por entonces, la Argentina enfrentaba una gran dificultad: la población del país había experimentado un crecimiento casi vegetativo. Entre 1810 y 1852 el incremento de sus habitantes fue tan sólo de 405.000 a 1.300.000. En este sentido, el aumento demográfico se presentaba como el motor económico y social para un país que necesitaba superar una forma política anacrónica y feudal, y que buscaba una organización democrática y una economía moderna. Este propósito adquiría categoría institucional a partir del artículo 25 de la Constitución de 1853 con el que se fomentaba la inmigración de los europeos ${ }^{2}$, en particular de aquellos que tuvieran por objeto labrar la tierra, mejorar las industrias e introducir las ciencias y las artes. Un año después empezaron a llegar las primeras familias europeas y se iniciaba así el proceso de inmigración masiva.

Culminado el período rosista, el entonces gobernador de Buenos Aires, Vicente López y Planes, determinó que hasta no contar con una organización definitiva de la Universidad, la Facultad de Medicina funcionaría al margen de aquella (Pérgola y Fustinoni, 1969, pp. 43-44). Así pues, su funcionamiento independiente se prolongó desde 1852 y por un período de poco más de veinte años. La pequeña elite médico-catedrática de entonces se ocupó de controlar exhaustivamente el acceso a la comunidad profesional de los egresados y nuevos médicos. Al tiempo que la Facultad se mantenía separada de la Universidad, su comunidad médica también se dividía entre la vieja dirigencia que ejercía el control sobre la orientación y los mecanismos de promoción institucionales, por un lado; y los jóvenes médicos y estudiantes, que cuestionaban esa dirigencia, por otro. Este grupo exigía reformas en los planes de estudio, en los reglamentos internos, en los sistemas de exámenes y en el monopolio de las asignaturas (Buchbinder, 2005, pp. 51-53; González Leandri, 1997, p. 33). 
En 1871, bajo el detonante del suicidio de un compañero de estudios «injustamente reprobado» se produjo la denominada revuelta estudiantil del 13 de diciembre (De Veyga, 1939, p. 14). Como portavoz de este movimiento juvenil en ascenso, José M. Ramos Mejía (1842-1914), por entonces estudiante del primer año de la carrera, protagonizó la empresa y cuestionó la imagen autocomplaciente de los catedráticos de la Facultad. Las publicaciones periódicas parecieron ser el medio para difundir sus denuncias, específicamente La Prensa editó sus artículos en los que plasmó las exigencias de los estudiantes. Como espacio de representación de la revuelta, un grupo de alumnos y jóvenes egresados crearon el Círculo Médico Argentino en 1873. Mediante este organismo, dirigido por Ramos Mejía, se ejerció una fuerte presión para que la Facultad fuera reincorporada a la Universidad de Buenos Aires. A través de la Revista Médico Quirúrgica (1864-1888), y luego de su propia publicación ${ }^{3}$, el Círculo Médico se ocupó de criticar el sistema de salud, basado en un criterio hospitalario y manejado por manos ajenas a la corporación médica (González Leandri, 1997, pp. 37-43; Buchbinder 2005, pp. 5253). Si bien las luchas políticas internas continuarían dentro de la Facultad de Medicina, se recuperaba finalmente la conexión con la Universidad.

Paralelamente, el proyecto para poblar el país resultaba marcadamente eficaz y los nuevos habitantes, que se centralizaron en las grandes ciudades, aumentaron de 1.300 .000 en 1859 a 7.885 .237 en

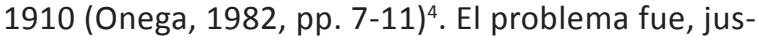
tamente, el defasaje entre el desmedido aumento demográfico y el limitado desarrollo estructural en una ciudad como Buenos Aires, que crecía caóticamente. Así pues, la inmigración masiva, la urbanización acelerada, los primeros y modestos desarrollos industriales dieron lugar a la problemática de la higiene y de la enfermedad en la ciudad, especialmente luego de las epidemias de cólera (1867-1868) y de fiebre amarilla $(1871)^{5}$. El problema higiénico se profundizó posteriormente a partir del arribo y el avance de otros brotes infecciosos (fiebre tifoidea, peste bubónica, viruela, sarampión, difteria, tuberculosis) y de los «males sociales» urbanos a ellos vinculados: el alcoholismo, la mala alimentación, la habitación insalubre, los excesos laborales y la prostitución. En consecuencia, los médicos higienistas adquirieron un protagonismo inusitado: colaboraban y aconsejaban en el trazado de espacios verdes en la ciudad, en la educación física de los niños y jóvenes, y en las recomendaciones sanitarias hacia los miembros de la comunidad.
Insoslayable fue, en esta coyuntura, la iniciativa de la pionera Revista Médico-Quirúrgica, que además de luchar contra el ejercicio ilegal de la medicina, debatía sobre tópicos como su enseñanza, la asistencia hospitalaria, el estado sanitario de la población, y las decisiones en torno a epidemias, vacunación, limpieza de las calles, reglamentación de la prostitución, ética médica, movimientos demográficos. Esta publicación colaboró tanto para que la esfera médica encontrara una dimensión fundamental de identidad, exaltación y reconocimiento, como para exponer la afirmación combativa de los ideales positivistas frente a las tradiciones religiosas que persistían en las instituciones de la salud (Vezzetti, 1985, pp. 29, 32).

Hacia los albores del siglo XX, más allá de la defensa del cuerpo físico de las personas amenazadas por microbios y bacterias se empezaba a hacer necesaria también la defensa social de un cuerpo demográfico amenazado por la «insalubridad» criminal, el otro mal moderno e invisible que también era necesario filtrar. Jorge Salessi (1995, pp. $23,115)$ señala el momento en que los inmigrantes empezaron a organizarse en asociaciones del movimiento obrero y a la huelga general de 1902, como las instancias en que los higienistas, hasta entonces preocupados por la amenaza de las enfermedades exóticas viajeras, comenzaron a inquietarse por la criminalidad, concebida como una enfermedad «psico-moral» que amenazaba la salud social. El efecto de continuidad entre ambas prácticas se sustentaba en similitudes metodológicas de los modelos de análisis y de la retórica de los médicos higienistas (de fines del siglo XIX) y las propias de los especialistas criminólogos (de principios del siglo XX), que colaboraron activamente en la imaginación y creación de nuevas obras de "higiene social», ahora destinadas a controlar el mal criminal.

Asimismo, en este período proliferaron una serie de instituciones psiquiátricas y criminológicas: la Oficina Antropométrica (1889) y el Servicio de Observación de Enfermos Mentales (1899) pertenecientes a la Policía Federal, la morgue dependiente del Instituto de Medicina Legal (1896), la Oficina Médico Legal dentro del Hogar Correccional de Menores (1905) y el Instituto de Criminología de la Penitenciaría Nacional (1907). Todo este proceso fundacional fue acompañado por las grandes publicaciones en el área como los Archivos de Psiquiatría, Criminología y Ciencias Afines, creados y dirigidos por José Ingenieros desde 1902 o la centenaria Semana Médica (1894-1994). 


\section{LETRAS MÉDICAS}

\subsection{Estudios sobre la locura en la Argentina: entre los casos clínicos y el Don Quijote}

Los pasos iniciales del higienismo en el país se desarrollaron de la mano del célebre médico argentino Eduardo Wilde (1844-1913). Wilde fue el primer presidente del Departamento Nacional de Higiene, creado en 1871. También se desempeñó, desde 1875, como profesor titular de las cátedras de Higiene (fundada dos años antes) y de Medicina Legal y Toxicología. En su tesis, titulada Disertación sobre el hipo (1870), Wilde ya anticipaba que "un conocimiento médico arraigado supone un saber profundo y una erudición suma (...) para ser médico se necesita recorrer los datos de la ciencias todas» (Wilde, [1870] 1923, p. 44).

Veinte años después, un Ramos Mejía ya médico, también renombrado higienista y profesor en la Cátedra de Enfermedades Nerviosas desde 1888, inspirado en la búsqueda de esta "una erudición suma», denuncia la difusión de una infundada creencia que por entonces circulaba en la Facultad de Medicina de la Universidad de Buenos Aires. Se trataba de la errónea opinión que sostenía que el médico perfecto debía «ignorar por completo las más rudimentales nociones de la educación literaria» (Ramos Mejía, 1893, p. 44). En su discurso ante el Círculo Médico Argentino, Ramos Mejía advierte sobre esta creencia instaurada por los viejos maestros de la carrera de medicina que creían que «el saber expresar con buenas formas sus ideas establece incompatibilidades con la clínica» (Ramos Mejía, 1893, p. 45). Al contrario, él enfatiza que la lectura es el vehículo ideal del médico para conseguir una expresión (escrita y oral) clara, así como para evitar «el desorden, la obscuridad, la incorrección» en sus explicaciones (Ramos Mejía, 1893, p. 46). Para culminar su argumento, Ramos Mejía apela a una serie de ejemplos por autoridad:

los grandes maestros son grandes escritores, como Bernard, Charcot, Trousseau, Duchenne (...) eminentes artífices del lenguaje encerrando dentro de su estilo grande, sólido y elegante, su pensamiento fuerte (Ramos Mejía, 1893, p. 46).

Los referentes franceses de las distintas especialidades de la medicina moderna son entonces recuperados como prototipos de esta exitosa imbricación entre el saber clínico y el saber oratorio y letrado.

Ahora bien, tanto Ramos Mejía como otros médicos del periodo, no sólo se refirieron a los beneficios expresivos que las prácticas de la educación literaria podrían otorgar a los alumnos, sino que ellos mismos articularon sus conocimientos letrados en el marco de sus explicaciones médicas. Así pues, en las Lecciones de Enfermedades Nerviosas y Mentales de Ramos Mejía son frecuentes las alusiones «a los tristes círculos del infierno del Dante» de La Divina Comedia, para sancionar moralmente a los alcohólicos delirantes en la «Lección VI: Los toxicómanos o bebedores de veneno» (Ramos Mejía, 1893, p. 176), o la indicación sobre el «amor neurasténico y desequilibrado de Madame Bovary» es considerada en la "Lección V: Los epilépticos bromiómanos» (Ramos Mejía, 1893, p 154). De este modo, referencias y ejemplos del saber letrado son incorporados en el marco de una lección académica. Sin embargo, será José Ingenieros (1877-1925), alumno de Ramos Mejía y luego profesor en distintas especialidades del área (Enfermedades Nerviosas, Medicina Legal, Psicología), quien recuperará de manera contundente el archivo literario en el desarrollo de sus explicaciones médico-psiquiátricas y criminológicas ${ }^{6}$.

Ingenieros escribe su tesis en medicina sobre "La simulación de la locura» en 1900 y un año después redacta un ensayo introductorio para tal obra, titulado "La simulación en la lucha por la vida». En este escrito, alude a múltiples ejemplos de la literatura occidental para ilustrar al arquetipo del simulador de enfermedades y del prototipo fraudulento propio de las grandes ciudades: «el Shylock, de Shakespeare; el Robert Macaire, de Lemaitre; el Mercadet, de Balzac; el Saccard, de Zola» (Ingenieros, [1901] 1954, p. 82). Plantea también que los mejores trabajos científicos no podrían mejorar las descripciones de modelos psicológicos tales como Macbeth, Stockmann, Saccard, Segismundo, Raskolnikoff, Sancho, es decir, las creaciones ficcionales de Shakespeare, Ibsen, Zola, Calderón, Dostoievsky o Cervantes, respectivamente (Ingenieros, [1901] 1954, p. 90).

Sobre este último autor en particular se detiene Ingenieros durante los capítulos de su tesis. En una de las secciones, titulada «Una página del Quijote», el médico explica a partir de la descripción del protagonista de la novela cómo el alienado real (a diferencia de un simulador de la locura) es irresponsable de sus acciones para la justicia. Ingenieros retoma el episodio en que el hidalgo hiere a los herreros que alteran su ceremonia de veneración de armas en la venta y recibe un posterior ataque por parte de aquellos. Ante esta situación, el ventero, "conocedor de la locura del Quijote» y de que "la locura es una causa eximente de pena» (Ingenieros, [1900] 1918, p. 119), lo defiende y ordena el cese del ataque ante los herreros ${ }^{7}$. 
Ahora bien, es importante destacar que la articulación de los documentos literarios en el desarrollo de un estudio científico no fue una propuesta originada entre los médicos argentinos, sino que se inscribe en una tradición europea que también había recurrido a este tipo de materiales para desarrollar distintas investigaciones, por ejemplo, en las áreas de la psiquiatría y de la medicina legal. En este orden, una sinopsis del informe sobre «Los degenerados y criminales en la obra de Zola» (1902) del criminólogo italiano Enrique Ferri fue publicada en los Archivos de Psiquiatría, Criminología y Ciencias Afines (desde ahora, Archivos). Ingenieros, que escribe la introducción del artículo, plantea que Ferri pudo ver en la figura del escritor naturalista francés Émile Zola al sociólogo detrás del novelista a partir del modo en que éste efectuó en su obra "una disecación de la sociedad» y «una profecía sobre los vicios y las miserias de la presente organización» (Ingenieros, 1902, p. 622).

En la misma revista se publica una investigación sobre "La locura de Don Quijote» (1906) de Antonio Rodríguez-Morini, psiquiatra español y Director de la Revista Frenopática Española. Rodríguez-Morini reconoce que Cervantes ha creado «el tipo más admirable de vesánico que pudiera apetecer como caso clínico el alienista más exigente» (Rodríguez-Morini, 1906, p. 763) y considera que así como la lectura del Quijote ha suscitado inagotables y magníficas reflexiones provenientes de disciplinas como la Gramática, la Historia, la Crítica y la Retórica, un papel similar debe ocurrir con las intervenciones proyectadas desde la Medicina Psicológica. La elaboración de RodríguezMorini dialoga de manera continua con el estudio «Primores del Don Quijote en el concepto médicopsicológico» (1886) del reconocido médico psiquiatra español Emilio Pi y Molist (1824-1892), también publicado en los Archivos (1912). Pi y Molist afirmaba que la enfermedad del personaje estaba de acuerdo con los conceptos de la monomanía admitidos por la medicina mental de entonces (Pi y Molist, 1912, pp. 611). En este sentido, la aceptación general que ofrecía este recurso interdisciplinar como aval para la reflexión científico-cultural no resultaba desconocida para los médicos argentinos que leían y publicaban en los Archivos. Este tipo de ejemplos, entonces, en los que el saber letrado invadía las explicaciones médicas, operaba también como punto de apoyo para afianzar los consejos concernientes a las ventajas otorgadas por la lectura, destinadas a los estudiantes. De hecho, contar con este tipo de saberes por parte de los alumnos facilitaba sus posibilidades de contextualizar las ejemplificaciones y, con ello, comprender las exposiciones.

\subsection{Médicos intelectuales y políglotas}

Más allá de las referencias de Ramos Mejía y de Ingenieros, la Revista del Centro de Estudiantes de Medicina (desde ahora RCEM) también se hizo eco de la preocupación por un saber más exhaustivo entre los futuros médicos. La RCEM se publicó desde septiembre de 1901, un año después de la fundación del renovado Centro de Estudiantes, hasta junio de 1909. Sobre todo en sus últimos números, y bajo la dirección del estudiante Héctor Taborda - que asumió el puesto de responsable principal con el número 83 en $1908^{8}$ - , el interés por la difusión de la literatura se constituyó en uno de los ejes primordiales de la RCEM. En distintas correspondencias dirigidas a los lectores, Taborda señala que la revista debe ser «la expresión de diversas modalidades intelectuales» y que sus columnas deben salirse del campo puramente científico para fomentar entre los estudiantes «la cultura de la letras», dado que "el caso clínico y los apuntes de las asignaturas ya no son, o no deberían ser, suficientes»; cada uno de los lectores además de ser estudiante de medicina «debería seguir el camino de otras aficiones intelectuales particulares» (Taborda, 1908, pp. 1-2). En este sentido, Taborda imagina la RCEM como un compendio que debe sumar: «el trabajo experimental o clínico», "el trabajo de abstracción», "la nota psicológica o sociológica» y «la contribución artística o jocosa» (Taborda, 1908, p. 2). En efecto, así fue y durante ese año la RCEM publicó poesías, cuentos y reflexiones literarias ${ }^{9}$. De este modo, Taborda recupera el mensaje de Ramos Mejía y de Wilde que convocaban a la necesidad de ampliar los conocimientos del alumnado, así como el concepto sobre un beneficioso desarrollo intelectual como resultado de una instrucción integral de saberes.

Como señalábamos, en junio de 1909 la RCEM dejó de publicarse como tal en tanto se produjo la asociación del Centro de Estudiantes con el Círculo Médico Argentino. De esta unión surgiría un nuevo órgano de difusión: la Revista del Círculo Médico y del Centro de Estudiantes de Medicina. Más de una década después, en 1922, Bernardo Houssay (1887-1971), por entonces profesor en la Cátedra de Fisiología, escribe en esta misma publicación un artículo sobre "La función de la Universidad». Houssay advierte sobre la carencia, en la Facultad de Medicina de Buenos Aires, de una atmósfera moral de camaradería, cortesía, lealtad y cariño, propia de las universidades inglesas y norteamericanas. Para ello, sugiere la necesidad de que los jóvenes «se interesen cada vez más por los deportes, los problemas filosóficos, el arte» (Houssay, [1922] 1989, p. 
231); así como señala el imperativo de mantener «un gran respeto y afecto por las actividades intelectuales y artísticas» (Houssay, [1922] 1989, p. 233).

Cuatro años después, en una conferencia sobre «La carrera de Medicina» ofrecida en el Colegio Nacional de Buenos Aires, Houssay invita a los estudiantes de la escuela secundaria a incursionar en los estudios médicos. Entre las aptitudes y ventajas que reivindica para abordar la carrera, señala el hecho de «dominar varios idiomas, pues abren nuevos campos de cultura; por lo menos traducir bien el francés y además, si es posible, el inglés o el alemán; en estos idiomas se aprende a traducir pronto y luego se dominan poco a poco con el ejercicio» (Houssay, [1926a] 1989, p. 37). En ese mismo año, en su discurso inaugural del curso de fisiología, "La fisiología y la medicina», Houssay plasma con referencias tal importancia, al citar en lengua original a Claude Bernard (uno de los médicos que algunos años antes Ramos Mejía refería como autoridad en el área): «ll faut prendre garde que les connaissances qui doivent armer l'intelligence ne l'accablent pas par leur poids» (Houssay, [1926b] 1989, p. 119), con el fin de enfatizar que el rol del profesor es el de orientar el aprendizaje de los alumnos, pero siempre respetando la independencia intelectual de cada uno ${ }^{10}$.

Además de citas como estas no fueron poco frecuentes las referencias en latín o en alemán ${ }^{11}$, así como la articulación de términos en inglés ("search and research», "full-time», "leader», "research professor», "research fellows») a lo largo de sus discursos. Aún más, en su conferencia titulada "La investigación científica», Houssay justifica la necesidad de implementar el término "research» que, sin tener su equivalente exacto en español, «literalmente significa una búsqueda incesantemente repetida o sea buscar y volver a buscar para aclarar cada vez mejor" (Houssay, [1943] 1989, p. 302)12.

Si bien Houssay apoyó el conocimiento y el manejo de idiomas por parte de los estudiantes y egresados con el propósito de abrir "nuevos campos de cultura», también lo hizo con el objetivo de facilitar el establecimiento de nexos con investigadores e instituciones extranjeras. Puede leerse en su obra, no sólo la atención puesta al desarrollo de la medicina y de su enseñanza en universidades inglesas, alemanas o norteamericanas (y la comparación entre estas y la de Buenos Aires), sino que además resulta recurrente la promoción del otorgamiento de becas para que los alumnos se formasen en distintas estadías en el exterior, así como el intercambio y la residencia temporal de profesores extranjeros.

\section{DEPORTE Y BUENA SALUD: LAS BASES DEL MÉDICO}

Desde los albores del siglo XX, la Facultad de Medicina de la Universidad de Buenos Aires ha intentado promover el desarrollo de las actividades físicas entre los estudiantes y futuros médicos. Las primeras motivaciones sobre tales prácticas se remontan, no obstante, hacia fines del siglo XIX, cuando una serie de conflictos limítrofes con Chile dispuso importantes preparativos bélicos preventivos: la convocatoria a la Guardia Nacional en 1892, la reorganización del Ejército Nacional dos años después y la refundación efectiva del Tiro Federal Argentino en 1895 (Bertoni, 1996, pp. 40-41). En efecto, los grupos militaristas pertenecientes al Ejército intervenían de manera firme en la programación tanto de la educación física como de las prácticas de gimnasia en las escuelas argentinas, entre las últimas décadas del siglo XIX y primeras del XX. Particularmente en aquellas escuelas coordinadas por el Consejo Nacional de Educación (que desde 1908 presidiría el mismo Ramos Mejía), los niños y los adolescentes recibían variados saberes, conductas disciplinarias y hábitos cotidianos concernientes a la tonificación de los cuerpos, a la prevención de las enfermedades y, sobre todo, al fortalecimiento físico a través de los regímenes de gimnasia militar y metodizada (Armus, 2007, p. 88; Bertoni, 1996, p. 54).

A partir del nuevo siglo, también la Facultad de Medicina se inscribió en el propósito de inculcar, en un alumnado no tan disciplinado como el escolar, los beneficios de ciertas actividades físicas vinculadas ya no a las prácticas de gimnasia pero sí a los deportes y el tiro. Así pues, en 1902 la RCEM publica los primeros avisos en torno a los concursos universitarios organizados por el Tiro Federal Argentino, y el Centro de Estudiantes efectúa variados certámenes previos para elegir a los mejores tiradores que representen a Medicina en el campeonato ${ }^{13}$. Vale aclarar que en esta década el Centro de Estudiantes de Medicina estrechó fuerte vínculos con la Liga Patriótica Argentina ${ }^{14}$. Así como la Liga apoyaba al Centro de Estudiantes en sus luchas y debates en torno a sus proyectos reformistas ${ }^{15}$, la RCEM, se ocupaba de difundir, entre otras actividades de la asociación, la convocatoria a los estudiantes universitarios a las peregrinaciones patrióticas por lugares históricos del país, cuya concurrencia implicaba el otorgamiento de diez mil pesos a cada universidad participante por parte del gobierno nacional.

Luego de los torneos de tiro, se conformó la Asociación Atlética de Medicina que durante la Tempo- 
rada Sportiva de 1906 participó en la Regata y en el Torneo Universitario. Este último, celebrado en el Club Gimnasia y Esgrima de Buenos Aires, constaba de Martillo, Bala, Cinchada, Carrera de 100, 200, 400 y 800 metros, Carrera de Vallas, Carrera de Postas, Salto en Alto, Salto en Largo, Football y Rugby. No obstante, la RCEM, aun antes de informar los resultados, mostró sus reparos frente a la escasa participación de los estudiantes:

[...] el número de representantes por Medicina fue muy reducido, pocos elementos nuevos, pues en su mayoría eran todos viejos conocidos de las lides sportivas universitarias. Esta indiferencia por los recomendables ejercicios al aire libre, es por demás lamentable. Su utilidad debe ser comprendida más que por nadie por los estudiantes de Medicina, que podrían obtener de ellos los mayores beneficios al conocer las reglas de la higiene moderna ${ }^{16}$.

Alejados ya del fomento de las actividades de tiro que buscaban despertar el sentimiento patriótico de los futuros médicos, en este momento los redactores de la RCEM articulan su recomendación de participación deportiva con el mensaje de la buena salud. Son los médicos y los estudiantes de medicina los que deben difundir, entre los habitantes de una sociedad jaqueada por las crisis higiénicas y epidémicas, sus saberes sobre las bondades que las actividades físicas ofrecen a una equilibrada salud. Dichos saberes se ligan al auge de las disciplinas higienistas que se establecieron en Buenos Aires a partir de las décadas de 1880 y 1890, como consecuencia de las nuevas problemáticas ligadas al hacinamiento en la ciudad y a los espacios laborales insalubres. Enunciados como "las bondades del verde urbano», "la respiración del aire puro», "el perjuicio de la palidez del encierro» y «la tonificación de los cuerpos» circulaban como los slogans más consensuados entre los médicos higienistas. Es en este sentido que la RCEM se inserta en la red de revistas que se ocupó de reflejar y propagar la inquietud sanitaria en los diferentes sectores de la sociedad, red de la que participaban publicaciones como La Argentina Médica, la Revista de la Liga contra la tuberculosis, los Anales de Sanidad Militar, el Eco Social de Barracas al Sud y Semana Médica ${ }^{17}$.

En tanto, la limitada respuesta de los futuros médicos por la práctica del fútbol -en la que Medicina «no había logrado reunir un núcleo de jugadores que la representaran $\aleph^{18}$ - pareció ampliamente compensada a partir de un repentino interés por el rugby, deporte que, en esta coyuntura, condensaba mayores virtudes que el balompié. De hecho, el desarrollo del fútbol, ya a nivel escolar, había despertado cierto malestar entre las filas de los médicos higienistas. En el mismo año del Torneo Universitario (1906), la revista La Higiene Escolar convocaba un concurso para que se premiara una nueva clase de ejercicios al aire libre que fuera capaz de sustituir al anacrónico y malsano fútbol, vehículo de corrupción moral, violencia corporal y cuestionables valores propios del mundo de la calle (Armus, 2007 , p. 94). El rugby, en cambio, representaba otros valores y la RCEM consideraba que cualquier hombre que lo practicara se daría cuenta de su superioridad frente al fútbol ${ }^{19}$, no sólo porque «una vez posesionado de las reglas del juego, en poco tiempo se puede llegar a ser un jugador discretamente bueno", sino también por «ser más viril» ${ }^{20}$.

Ahora bien, el propósito de alentar la práctica de estas actividades deportivas no iba a permanecer ajeno al interés de otras revistas médicas del período, ni tampoco se manifestaría como una inquietud meramente local. En su sexto ciclo, los Archivos publican el artículo "La educación física en las universidades» del médico fisiólogo italiano Angelo Mosso (1846-1910). El texto de Mosso da cuenta de las diferencias entre la vida deportiva de los estudiantes de medicina en Inglaterra y Estados Unidos, frente a los alumnos de universidades italianas, francesas e incluso alemanas. En los primeros casos, la actividad física se presenta como intensiva en la rutina de los estudiantes, quienes al momento de inscribirse son vistos «primeramente por el médico y el maestro de gimnasia, los cuales les indican y aconsejan la clase de vida y los ejercicios que deben hacer, y al cabo de un año se anotan los resultados obtenidos» (Mosso, 1905, p. 369). Mosso entiende que es tal la estima por la educación física en estas culturas que aún los rectores y profesores secundan la propaganda a favor de los juegos. En cambio, en las escuelas de medicina de las Universidades de Berlín, París o Roma la educación física se describe como "descuidada» y los alumnos resultan no sólo "más débiles y afeminados» que sus colegas angloparlantes, sino que también son «más propicios al vicio» (Mosso, 1905, pp. 368-369). En un sentido similar a la RCEM, el artículo de los Archivos remite a la representación de una vinculación entre actividad física y masculinidad y remarca el modo en que los deportes hacían de los estudiantes hombres más "disciplinados» y "valerosos» (Mosso, 1905, p. 372), pero además enfatiza la relación consecutiva entre la salud física y la habilidad intelectual, en tanto explica cómo el ejercicio de los músculos procuraba «una condición esencial para el desenvolvimiento de los órganos de la inteligencia» así como «las funciones del cerebro se desarrollaban mejor gracias al equilibrio físico» (Mosso, 1905, p. 371). 
Como habíamos señalado, en «La función de la Universidad» Houssay también se refiere a la importancia de que los jóvenes de la Facultad de Medicina participen en las prácticas deportivas, además de estudiar cuestiones filosóficas y artísticas, más allá del espectro de conocimientos médicos. En su caso, el elemento que justificó la necesidad de una actividad física entre los estudiantes se alejó de la exaltación de un sentimiento patriótico nacional. Sí fueron recuperadas, en cambio, las máximas que vinculaban el deporte con la buena salud y su necesidad para fortalecer a aquellos que se dispusieran a afrontar estudios tan arduos como la medicina supone, por un lado, pero también para afianzar el sentimiento de confraternidad que estas actividades tienden a generar entre los compañeros. En la conferencia sobre «La carrera de Medicina», Houssay advierte que "una buena salud física es casi indispensable en una carrera fatigosa al que se está expuesto a contagios. Los jóvenes débiles o de salud ya afectada no conviene que estudien medicina» (Houssay, [1926a], 1989, p. 36). Vale aclarar en este punto que Houssay estaba en una cruzada por la limitación de cupos para los alumnos de medicina en Buenos Aires. Durante la década de 1920 se registraba en la Argentina un crecimiento en la matrícula de las distintas casas de estudios superiores. Como Consejero de la Facultad, Houssay promovió una Ordenanza al Consejo Directivo de Medicina, a fines de 1926, que se proponía limitar cuantitativamente el ingreso de estudiantes a la Facultad (Cibotti, 1996, p. $45)^{21}$. En distintos discursos de Houssay puede leerse el modo en que alentaba a realizar estos estudios a los jóvenes comprometidos, voluntariosos, responsables, saludables y altruistas, y paralelamente desalentaba a aquellos que no respondieran a tales características. En este sentido, las recomendaciones por la práctica de actividades físicas como motivadoras de la buena salud, en un momento iniciático para los alumnos, son recuperadas para estimular conductas saludables entre los jóvenes, pero también serán re-direccionadas para discriminar entre candidatos "aptos» y candidatos «débiles» al inicio de la carrera.

\section{DE LA FACULTAD DE MEDICINA A LA CIUDAD UNI- VERSITARIA}

Para 1940, Houssay presenta una ponencia en el Congreso del Profesorado Argentino (Buenos Aires), luego publicada en la Revista Médica de Córdoba, sobre la «Función social de la Universidad». Interesado en una problemática más general sobre los saberes que circulan en las universidades, y ya no sólo sobre aquellos propios de la Facultad de Medicina, Houssay postula que las escuelas fundamentales de toda Universidad deben ser: Filosofía, Ciencias y Letras (Houssay, [1940] 1989, p. 235). Entiende que en una institución como la Academia, donde se debe cultivar la universalidad de los conocimientos humanos, son fundamentales las disciplinas desinteresadas y básicas que buscan el amor a la verdad y la educación de la inteligencia.

En este marco, y teniendo como ejemplos instituciones extranjeras, Houssay confía en el ideal de una ciudad universitaria, o de un campus, para Buenos Aires. La ciudad universitaria, lejana de una mera continuidad de edificios, suponía para el médico beneficios como «el comercio intelectual frecuente», "la convivencia con hombres selectos y de conocimientos variados, unidos por ideales comunes, en un ambiente elevado donde florecen capacidades creadoras de los estudiosos» (Houssay, [1940] 1989, p. 242). Las ciudades universitarias fomentan la cooperación y el clima de ideas, y se constituyen de este modo en el ámbito ejemplar donde Houssay concibe los estudios: un espacio donde los alumnos se instruyan en una atmósfera de cultura general y tengan la posibilidad de realizar cursos de otras Facultades y enriquecer, de esta manera, sus capitales intelectuales. En fin, «las ciudades universitarias permiten que en un ambiente social, cultural y moral elevado, se desarrollen y eduquen en forma integral las aptitudes del hombre: intelectuales, estéticas, morales y físicas» (Houssay, [1940] 1989, p. 242); las imaginaba Houssay como «ciudades universitarias que tendremos alguna vez como todos los pueblos más adelantados, y que ya ha planeado el Brasil en Sud América» (Houssay, [1940] 1989, p. 241).

Lo cierto es que recién en 1962 comenzaría la construcción del Primer Pabellón de la Ciudad Universitaria en el barrio de Núñez, al extremo norte de la Ciudad de Buenos Aires. En este edificio tuvieron lugar los departamentos de Física, Matemática y Meteorología de la Facultad de Ciencias Exactas y Naturales. Para 1971 ya se habían edificados los Pabellones II y III que albergaban (y aún hoy lo hacen) a los Departamentos de Química, Biología y Geología de Ciencias Exactas y Naturales, y a la Facultad de Arquitectura y Urbanismo. Si bien existían los planos para construir, al menos, dos pabellones más, las obras quedaron inconclusas y jamás se concretaron. La Facultad de Medicina de la Universidad de Buenos Aires, como otras casas de estudio de esta institución, nunca llegó a ser parte de la Ciudad Universitaria. El concepto de campus modélico, como aquella agrupación de institutos, facultades, bibliotecas, habitaciones y sitios pensados para el sano esparcimiento que debían facilitar la convivencia y el intercambio frecuente de ideas entre los 
alumnos que cultivan las distintas ramas del saber, no se vería entonces concretado para la formación integral (intelectual, ética, física, estética y social) del estudiante de medicina.

\section{CONCLUSIONES}

En fin, este recorrido por algunas voces y discursos de la medicina argentina intentó mostrar ciertas inquietudes acerca de la formación de los estudiantes entre 1870 y 1940 . Discursos, tal vez menos cuantitativamente numerosos que cualitativamente representativos - dada la impronta de sus voceros en los avances de la ciencia y la cultura nacional-, que buscaron proponer un perfil de médico que fuera capaz de combinar sus conocimientos específicos con otros de la cultura letrada, pero también con los hábitos de una buena condición física, implicados ambos (de una u otra manera) en beneficio de su carrera.

El interés por tal condición física fue promovido, por un lado, a partir de distintas instancias de motivación: el deporte como signo de salud, el compromiso patriótico del alumnado, la promoción de la actividad al aire libre como medio de difusión del mensaje higienista, el ensayo de estas prácticas para consolidar la camaradería y la virilidad, o el apto estado físico tanto para alcanzar un desarrollo intelectual acorde, como para encarar un estudio de las magnitudes que supone la carrera de medicina. Como fuese, lo cierto es que el mensaje circuló durante estas décadas y, hasta donde sabemos, no fue recibido ni asimilado por los destinatarios con el mismo énfasis con el que fue propuesto por sus emisores. Por otro lado, en tanto todo médico debía saber expresarse con claridad, fluidez y sabiduría, la cultura de las letras también fue buscada entre los estudiantes y se apoyaba, en este caso, en otros valores y virtudes: las ventajas retóricas y oratorias para mejorar las explicaciones científicas, el conocimiento de idiomas para fomentar el vínculo con el extranjero y la recepción de fuentes internacionales, pero también la lectura de las obras literarias fue estimulada para aprender a partir de la riqueza descriptiva de los más célebres cuadros patológicos de los personajes de ficción.

Si bien muchas de estas propuestas han quedado relegadas o perimidas entre los proyectos de las asignaturas que conforman en la actualidad la carrera de medicina en la Universidad de Buenos Aires, consideramos pertinente su recuperación y su relectura, casi un siglo después en algunos casos, con el fin de reflexionar acerca de los saberes o habilidades que fueron demandadas a los futuros médicos en distintos momentos de la historia de esta institución en la Argentina.

\section{NOTAS}

1. Militar argentino, representante de la aristocracia rural, Juan Manuel de Rosas (1793-1877) dirigió políticamente el país desde la entonces denominada Confederación Argentina, entre 1835 y 1852 . Se trata de una figura controversial, considerada, por sus detractores -entre ellos muchos intelectuales del período- como un tirano despótico e intolerante, instigador del terror de Estado, la tortura y la muerte; y por sus admiradores, como un líder de los sectores populares (Salvatore, 1998, pp. 325-326, 331).

2. Si bien la necesidad de población laboralmente activa no debía distinguir origen, el gobierno entendía la conveniencia de atraer europeos dado que por razones culturales y geográficas podían incorporarse al nuevo esquema de progreso sin mayores inconvenientes (Onega, 1982, p. 10).

3. Los Anales del Círculo Médico se fundaron en 1877, bajo la dirección de Ramos Mejía y se publicaron de manera independiente hasta el año 1909, momento en que se produjo la unión del Círculo con el Centro de Estudiantes de Medicina.

4. En términos de procedencias es certero afirmar que hasta 1910 se radicaron alrededor de 1.000 .000 de italianos (en especial, piamonteses, genoveses, napolitanos, calabreses), 700.000 españoles (gallegos, vascos, andaluces),

90.000 franceses, 70.000 rusos, en su mayor parte de origen judío, 65.000 turcos, 35.000 austro-húngaros, 20.000 alemanes y un número inferior de portugueses, suizos, ingleses, belgas y holandeses. A partir de 1910 empezó a disminuir la afluencia de italianos (que a comienzos del siglo XX representaban el $45 \%$ total de inmigrantes), y se multiplicó, en cambio, la llegada de españoles que hasta 1920 conformaron la mitad de los recién llegados.

5. La epidemia de fiebre amarilla llevó a la muerte a trece mil personas (el $8 \%$ de la población) y aceleró el éxodo masivo, iniciado en la década del 50 , de la elite porteña hacia la zona norte de la ciudad.

6. Además, Ingenieros se desempeñó como Director del Servicio de Observación de Alienados, hacia 1904, y como Director del Instituto de Criminología de la Penitenciaría Nacional desde su fundación en Buenos Aires en el año 1907.

7. En efecto, una de las consideraciones que sostuvo Ingenieros en su tesis, y por lo que combatió contra las anacrónicas leyes del período que rechazaban su reformulación, fue la que sostenía los criterios de responsabilidad legal del delincuente y de irresponsabilidad del alienado. 
8. Taborda fue un estudiante paradigmático en tanto representante de la Universidad de Buenos Aires en distintos congresos latinoamericanos que trataron la problemática sobre la reforma universitaria (Biagini, 2006, p. 84). En el marco de la Facultad de Medicina fue adscripto de José Ingenieros, cuando éste ocupaba el puesto de Director en el Instituto de Criminología.

9. En orden a estos propósitos, el número 88 de la RCEM publica las estrofas de La Maison Ideale -en lengua originaldel poeta francés Xavier Privas (1908, pp. 310-311), y en el volumen 93 edita el poema Le prodige del escritor y político parisino Pons de Verdun (1909, p. 250). En su último número, en tanto, la RCEM presenta, "El manuscrito de un médico de aldea", cuento de Anatole France (n. 94, 1908, pp. 92-100), entre otros.

10. La cita refiere; "Hay que tratar de evitar que los conocimientos que deben armar la inteligencia no terminen por destruirla".

11. Por un lado, en su escrito sobre "Problemas y orientaciones de la medicina moderna", Houssay señala que el rol de un profesor universitario de clínica es el de coordinador entre colaboradores, un primum inter pares" (Houssay, [1927] 1989, p. 48). En tanto, en su reflexión sobre "Claude Bernard y el método experimental" explica, también en latín, las reglas del método comparativo: "posita causu ponitur effectus" (el experimento); "oexperimentum crucis (sullata causa tollitur effectus)" (la contraprueba); "variante causa variatur effectus" (el experimento comparativo) (Houssay, [1941] 1989, p. 455). Por otro lado, en el artículo "El descubrimiento de la diabetes pancreática", Houssay cita en alemán y traduce partes de los hallazgos de Josef von Mering y Oskar Minkowski sobre el tema, referidos por el profesor Naunyn en su libro Erinnerungen, Gedaken und Meinungen, así como las cartas entre Minkowski y Thierfelder, un discípulo de von Mering (Houssay, [1955] 1989, pp. 471-482).

12. El uso del inglés, en la discursividad houssayana no se limitó a la articulación de términos. El fisiólogo escribe en esta lengua, "The role of the Hypophysis in Carbohydrate Metabolism and in Diabetes", su conferencia Nobel pronunciada en Estocolmo (Houssay, [1947] 1989, pp. 192-198), así como su artículo "Trends in Physiology as seen from South America" publicado en el Annual Review of Physiology de 1956 (Houssay, [1956] 1989, pp. 199-211).

13. Comisión Redactora (1902), "Tiro Federal Argentino. Concursos Universitarios", RCEM, 11, p. 317; Comisión Redactora (1902), "Tiro Federal Argentino. Concursos Universitarios", RCEM, 12, p.349.
14. Se trata de la asociación fundada en la Argentina en el año 1898 y cuyo fin era el de estimular y vigilar cualquier instancia relacionada con la inminente situación de guerra con Chile. Estaba constituida por un grupo de funcionarios aglutinados en torno a la defensa nacional. Su principal preocupación fue la de crear una red de propaganda patriótica integrada por las filiales que la misma Liga había fundado en cada una de las ciudades y pueblos de la República, además de propiciar la construcción de polígonos de tiro que extendieran la instrucción militar al conjunto de la población (Bertoni, 1996, pp. 53-54).

15. Junta Ejecutiva de la Liga Patriótica (1906), “Nota de la Liga Patriótica", RCEM, 56, pp. 398-399.

16. Comisión Redactora (1906), "Reseña de la actuación de los elementos de la Asociación Atlética de Medicina durante la temporada Sportiva de 1906", RCEM, 56. pp. 431-432.

17. Comisión Redactora (1901), "Programa de Higiene", RCEM, 2, p. 50; Comisión Redactora (1903), "Cátredra de Higiene", RCEM, 3, p. 78; Comisión Redactora (1904), "La Higiene y la Facultad", RCEM, 31, p. 813; Comisión Redactora (1906), "Reseña de la actuación de los elementos de la Asociación Atlética de Medicina durante la temporada Sportiva de 1906", RCEM, 56. p. 430.

18. Comisión Redactora (1906), "Reseña de la actuación de los elementos de la Asociación Atlética de Medicina durante la temporada Sportiva de 1906", RCEM, 56, p 432.

19. Desde su apogeo a fines del XIX, el fútbol se emplazó en los más distantes lugares de la sociedad porteña, instalándose en toda suerte de potreros, sobre todo en las zonas bajas aledañas al Río de la Plata; a diferencia del rugby o el tenis, deportes exclusivos que había cultivado la comunidad británica, y que hacia 1900 se habían incorporado a las prácticas de las familias criollas acomodadas (Barrancos, 2000, pp. 597-598).

20. Comisión Redactora (1906), “Reseña de la actuación de los elementos de la Asociación Atlética de Medicina durante la temporada Sportiva de 1906", RCEM, 56, p 432.

21. Según la perspectiva de Alfonso Buch, el propósito de Houssay con estos señalamientos sobre la posibilidad de dedicarse a otras tareas intelectuales, artísticas o deportivas era, justamente, el de convencer a los estudiantes de que existían otras carreras alternativas a la medicina y el de intentar explicarles el sinsentido que implicaba el aumento de médicos en ejercicio en el país (Buch, 2006, p. 277). 


\section{BIBLIOGRAFÍA}

Armus, Diego (2007), La ciudad impura. Salud, tuberculosis y cultura en Buenos Aires, 1870-1950. Buenos Aires, Edhasa.

Barrancos, Dora (2000), “La vida cotidiana”. En: Lobato, Mirtha (dir.), Nueva Historia Argentina. El progreso, la modernización y sus límites (1880-1916), Buenos Aires, Sudamericana.

Bertoni, Lilia (1996), "Soldados, gimnastas y escolares. La escuela y la formación de la nacionalidad a fines del siglo XIX", Boletín del Instituto de Historia Argentina y Americana Dr. Emilio Ravignani, 13, pp.35-57, p. 40, 41, 53, 54.

Biagini, Hugo (2006), “Redes estudiantiles en el Cono Sur (19001925)". En: Marsiske, Renate (Coord.), Movimientos Estudiantiles en la historia de América Latina, Vol III, Ciudad de México, Plaza y Valdés Editores.

Cibotti, Ema (1996), "Bernardo Houssay y la defensa de la Universidad científica en Argentina", Estudios Interdisciplinarios de América Latina y el Caribe, 7 (1), pp. 41-55.

Buch, Alfonso (2006), Forma y función de un sujeto moderno. Bernardo Houssay y la fisiología argentina (1900-1943). Buenos Aires, Universidad Nacional de Quilmes Editorial.

Buchbinder, Pablo (2005), Historia de las Universidades Argentinas. Buenos Aires, Sudamericana.

González Leandri, Ricardo (1997), “Académicos, doctores y aspirantes. La profesión médica y la reforma universitaria: Buenos Aires 1871-1876", Entrepasados, Año IV (12), Buenos Aires, pp. 31-54, p. 37, 39, 42, 43.

González Leandri, Ricardo (2001), “Notas acerca de la profesionalización médica en Buenos Aires durante la segunda mitad del siglo XIX". En: Suriano, J. (comp.) La cuestión social en la Argentina, 1870-1943, Buenos Aires, La Colmena.

Houssay, Bernardo (1989 [1922]), “La función de la Universidad". En: Barrios Medina, A. y Paladini, A. (comps.) Escritos y discursos del doctor Bernardo Houssay, Buenos Aires, Eudeba.

Houssay, Bernardo (1989 [1926a]), "La carrera de medicina". En: Barrios Medina, A. y Paladini, A. (comps.) Escritos y discursos del doctor Bernardo Houssay, Buenos Aires, Eudeba.

Houssay, Bernardo (1989 [1926b]), "La fisiología y la medicina". En: Barrios Medina, A. y Paladini, A. (comps.) Escritos y discursos del doctor Bernardo Houssay, Buenos Aires, Eudeba.

Houssay, Bernardo (1989 [1927]), "Problemas y orientaciones de la medicina moderna". En: Barrios Medina, A. y Paladini, A. (comps.) Escritos y discursos del doctor Bernardo Houssay, Buenos Aires, Eudeba.

Houssay, Bernardo (1989 [1940]), "Función social de la Universidad". En: Barrios Medina y Paladini (comps.) Escritos y discursos del doctor Bernardo Houssay, Buenos Aires, Eudeba.
Houssay, Bernardo (1989 [1941]), “Claude Bernard y el método experimental". En: Barrios Medina, A. y Paladini, A. (comps.) Escritos y discursos del doctor Bernardo Houssay, Buenos Aires, Eudeba.

Houssay, Bernardo (1989 [1943]), "La investigación científica". En: Barrios Medina, A. y Paladini, A. (comps.) Escritos y discursos del doctor Bernardo Houssay, Buenos Aires, Eudeba.

Houssay, Bernardo (1989 [1947]), "The role of the Hypophysis in Carbohydrate Metabolism and in Diabetes". En: Barrios Medina, A. y Paladini, A. (comps.) Escritos y discursos del doctor Bernardo Houssay, Buenos Aires, Eudeba.

Houssay, Bernardo (1989 [1955]), "El descubrimiento de la diabetes pancreática". En: Barrios Medina, A. y Paladini, A. (comps.) Escritos y discursos del doctor Bernardo Houssay, Buenos Aires, Eudeba.

Houssay, Bernardo (1989 [1956]), "Trends in Physiology as seen from South America". En: Barrios Medina, A. y Paladini, A. (comps.) Escritos y discursos del doctor Bernardo Houssay, Buenos Aires, Eudeba.

Ingenieros, José (1918 [1900]), La simulación de la locura, 8a ed. Buenos Aires, L. J. Rosso.

Ingenieros, José (1954 [1901]), La simulación en la lucha por la vida. Buenos Aires, Meridion.

Ingenieros, José, (1902), "Introducción a Los Degenerados y Criminales en la obra de Zola de Enrique Ferri", Archivos de Psiquiatría, Criminología y Ciencias Afines, I, pp. 622- 670.

Mosso, Angelo (1905), "La educación física en las universidades", Archivos de Psiquiatría, Criminología y Ciencias Afines, VI, pp. 368-372.

Onega, Gladys (1982), La inmigración en la literatura argentina (1880-1910). Buenos Aires, Centro editor de América Latina.

Pérgola, Federico y Fustinoni, Osvaldo (1969), La Facultad de Medicina de Buenos Aires y otros temas de la historia de la Medicina. Buenos Aires, Editorial Macchi.

Pi y Molist, Enrique (1912), "Primores del Don Quijote en el concepto médico-psicológico", Archivos de Psiquiatría, Criminología y Ciencias Afines, XI, pp. 611-616.

Ramos Mejía, José (1893), "Discurso ante el Círculo Médico". En: Estudios clínicos de enfermedades nerviosas y mentales, Buenos Aires, Félix Lajouane.

Ramos Mejía, José (1893), “Lección V: Los epilépticos bromiómanos". En: Estudios clínicos de enfermedades nerviosas y mentales, Buenos Aires, Félix Lajouane.

Ramos Mejía, José (1893), “Lección VI: Los toxicómanos o bebedores de veneno". En: Estudios clínicos de enfermedades nerviosas y mentales, Buenos Aires, Félix Lajouane. 
Rodríguez Morini, Antonio (1906), "La locura de Don Quijote", Archivos de Psiquiatría, Criminología y Ciencias Afines, Año V, pp. 763-767.

Salessi, Jorge (1995), Médicos maleantes y maricas. Buenos Aires, Beatriz Viterbo.

Salvatore, Ricardo (1998), “Consolidación del régimen rosista (1835-1852)". En: Goldman, N. (Dir.), Nueva Historia Argentina. Revolución, República, Confederación (1806-1952), Buenos Aires, Editorial Sudamericana.
Taborda, Héctor (1908), “Carta a mis lectores", Revista del Centro de Estudiantes de Medicina, 83, pp. 1-2.

Veyga, Francisco de (1939), “Vida y trabajos del Dr. José María Ramos Mejía", Semana Médica, 45, Buenos Aires, pp. 31, p. 14.

Vezzetti, Hugo (1985), La locura en la Argentina. Buenos Aires, Paidós.

Wilde, Eduardo (1923 [1870]), “Disertación sobre el hipo". En: Obras completas, Buenos Aires, Peuser. 